\title{
Systematics of isotope ratio measurements with resonant laser photoionization sources
}

\author{
R.K. Wunderlich*, I.D. Hutcheon, G.J. Wasserburg and G.A. Blake \\ Division of Geological and Planetary Sciences, California Institute of Technology \\ Pasadena Ca 91125, \\ *Physikalisches Institut, University of Augsburg, 8900 Augsburg FRG
}

\begin{abstract}
Sources of laser-induced even-even and odd-even isotopic selectivity in the resonance ionization mass spectroscopy of Os and Ti have been investigated experimentally for various types of transitions. A set of conditions with regard to laser bandwidth and frequency tuning, polarization state and intensity was obtained for which isotopic selectivity is either absent or reduced below the $2 \%$ level.
\end{abstract}

\section{INTRODUCTION}

Resonance Ionization Mass Spectrometry (RIMS) in combination with ion microbeam sources has been successfully applied to the in situ trace element analysis of many elements ${ }^{1,2}$. The high sensitivity of RIMS for elemental analysis arises from the elemental selectivity of the resonant photoionization process ${ }^{3}$ which allows mass spectrometry at low mass resolving power and high transmission, the high efficiency of the photoionization process and the fact that neutral atoms dominate the flux of sputtered particles. These characteristics make RIMS, in combination with ion sputtering sources potentially a very important tool for the in situ isotopic analysis of meteoritic samples in which isotopic anomalies resembling contributions of distinct stellar sources, and localized in relic interstellar grains, are observed on a very small spatial scale.,5 The use of RIMS for precise isotopic analysis is, however, complicated by shifts in the apparent isotope ratios which are intrinsic to the resonant photoionization process. Observed shifts in isotope ratios range from $3 \%$ to almost $100 \%,{ }^{6,7,8,9,10}$ typically with an enhancement of the odd isotopes (odd - even effect). In a recent RIMS analysis of Ti by Spiegel $e t$ al. ${ }^{10}$ laser-induced shifts of up to $46 \%$ were observed with a consistent enrichment of the odd isotopes. These observations raise questions as to the physical sources of the observed behavior ${ }^{11,12,13}$ and whether methods to reduce or totally avoid laserinduced isotopic selectivity in RIMS can be developed. ${ }^{11,13,14}$ In principle it is possible to correct measured isotope ratios by comparison with standards of known isotopic composition. However, the size of the observed laser-induced effects introduces a large uncertainty in isotopic compositions obtained in this way and impedes the ready identification of samples of unusual isotopic composition.

We report here on the recent results of a RIMS study of Os and Ti isotope ratios in which we experimentally investigated sources of laser-induced isotopic shifts and devised methods to reduce these effects below the $1 \%$ level. ${ }^{14,15}$ Os and $\mathrm{Ti}$ were chosen because of their importance in geo - and cosmochemistry.

We broadly distinguish three sources of laser-induced isotopic selectivity in RIMS: (i) laser bandwidth and tuning effects, (ii) dynamic effects and (iii) selection rule and polarization effects. The experimental and theoretical investigation of these effects provides constraints on the parameters of the resonant 
photoionization process such as laser bandwidth, accuracy of wavelength tuning, laser power and polarization state and the choice of a particular ionization scheme which allow, under selected conditions, measurement of isotope ratios differing from the true abundances only by pure mass fractionation.

\section{EXPERIMENTAL SET-UP}

A single colour one photon resonant ionization scheme with resonance states in the region between 38000 $\mathrm{cm}^{-1}$ and $40500 \mathrm{~cm}^{-1}$ was applied to the RIMS analysis of Ti and Os. ${ }^{14}$ The laser system consisted of a $200 \mathrm{~Hz}$ rep. rate excimer-laser-pumped dye laser equipped with a BBO crystal for frequency doubling. The dye laser could be operated with an intracavity etalon and in some wavelength regions also in a lower grating order. This allowed measurements with laser bandwidths of $0.04 \mathrm{~cm}^{-1}, 0.20 \mathrm{~cm}^{-1}$ and $0.35 \mathrm{~cm}^{-1}$ in the fundamental output. Low bandwidth operation is important to obtain an upper limit on the width of the hyperfine transition array of the odd isotopes, while in the large bandwidth mode wavelength dependence of measured isotope ratios can be greatly reduced. The mass analyzer was a modified Finnegan MAT thermal ion source quadrupole mass spectrometer. Ti and Os samples were prepared by alloying the metal powder with a tantalum filament ribbon. The beam of neutral atoms from the heated filament was intersected by the laser beam and ionized. Photoions were detected with a $80 \mathrm{MHz}$ counting system, which allowed the measurement of up to 40 ions per laser pulse without significant dead time correction. ${ }^{14}$ The absence of isoboric interferences was experimentally verified in all experiments.

\section{WAVELENGTH TUNING AND BANDWIDTH EFFECTS}

In the presence of optical isotope shifts (IS) and hyperfine structure (HFS) measured isotope ratios can depend on the laser wavelength setting, bandwidth and intensity due to an incomplete overlap of the laser spectral distribution with the absorption maxima of the individual isotopes. This dependence is more pronounced for thermal atom sources compared to sputtered secondary neutral sources because of the much larger Doppler width for the latter. With the exception of wavelength tuning effects it is expected that the physical effects responsible for laser-induced isotope selectivity are the same for thermal ion sources and secondary neutral sources. In addition, the RIMS process with thermal atom sources has a higher selectivity due to the absence of sputtered excited molecules, which allows the application of simple ionization schemes. For quantitative isotopic analysis it is important to establish criteria which uniquely relate the ratios of measured ion signals at masses $\mathrm{i}$ and $\mathrm{j}, R_{M}^{i j}$, to the true abundance ratio in the sample. ${ }^{16,17}$ This requires that wavelength tuning effects can be separated from pure mass fractionation. Here we focus on the even isotopes because of the absence of any intrinsic odd-even effects, which can only be quantified if wavelength tuning effects are absent or can be corrected analytically.

Pure mass fractionation can be characterized by a fractionation law which relates $R_{M}^{i j}$ to the value of a known reference standard:

$$
R_{M}^{i j} \cdot f(\alpha)=R_{S}^{i j}
$$

The fractionation factor $f(\alpha)$ is an empirical function of the form $(1+n \alpha),(1+\alpha)^{n}$ or $\exp (\alpha n), \alpha$ is the fractionation per unit mass and $n=i-j$. Isotope ratios measured by RIMS are independent of wavelength tuning effects only if laser parameters can be established for which $\alpha$ is constant for all even isotope pairs, independent of mass, and is unaffected by changes in laser detuning and bandwidth.

Differences in the ionization probability of even isotopes arise from the dependence of the effective cross section for the discrete absorption, $\sigma_{A}^{i}$, on the detuning, $\Delta_{i}$, of the laser center wavelength from the 


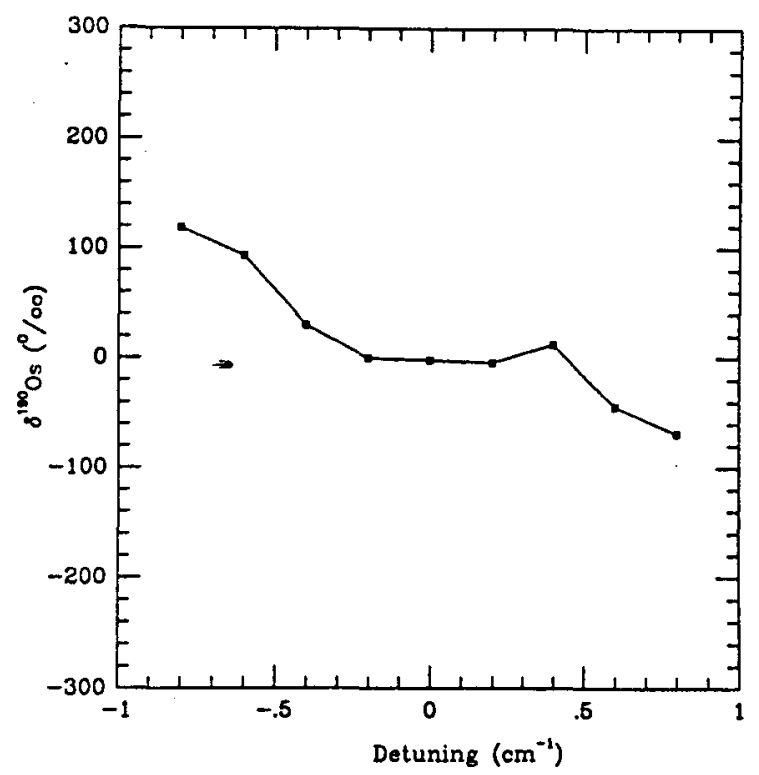

Figure 1. Wavelength dependence of the ${ }^{190} \mathrm{Os} /{ }^{188} \mathrm{Os}$ isotope ratio ( $\delta$-values in permil relative to standard reference value ${ }^{18}$ measured for the $39407 \mathrm{~cm}^{-1}, \mathrm{~J}=5$ resonance state. $\Delta \omega_{\mathrm{L}}=0.70 \mathrm{~cm}^{-1}$. Typical $1 \sigma$ error of data points $1-1.5 \%$

absorption maxima of the individual isotope. The discrete resonance is saturated if $\mathrm{F}_{\mathrm{L}} \times \sigma_{\mathrm{A}} \times \mathrm{t}_{\mathrm{p}}>1$, where $F_{L}$ and $t_{P}$ are total photon flux and laser pulse duration, respectively. If the condition $F_{L} \times \sigma_{A} \times t_{P}>1$ is met for isotopes $i$ and $\mathrm{j}$ in some tuning interval of the laser, then the ratio $R_{M}^{i j}(\lambda)$ will be constant over that interval. Thus the condition $R_{M}^{i j}(\lambda)=$ const. establishes an isotope ratio which can be considered to differ from the true ratio in the sample only by pure mass fractionation. This is demonstrated in Fig. 1 where the wavelength dependence of the ${ }^{190} \mathrm{Os} /{ }^{188} \mathrm{Os}$ ratio, expressed as the per mil deviation from the standard value ${ }^{18}$ is shown. In this experiment $\Delta \omega_{\mathrm{L}} / \Delta=10$, where $\Delta \omega_{\mathrm{L}}$ is the laser bandwidth and $\Delta \mathrm{T}$ is the optical isotope shift between ${ }^{190} \mathrm{Os}$ and ${ }^{188} \mathrm{Os}$. Model calculations, which exclude saturation effects ${ }^{19}$ do not predict $R_{M}^{i j}(\lambda)=$ const. for $\Delta \omega_{L} / \Delta \mathrm{T}=10$, emphasizing the role of power broadening. The width of the tuning interval for which $R_{M}^{i j}(\lambda)=$ const. directly shows the accuracy of laser wavelength setting required to obtain reproducible isotope ratios. The value of $R_{M}^{i j}$ established in the region $R_{M}^{i j}(\lambda)=$ const. can serve as an internal wavelength standard for laser wavelength tuning. Using ${ }^{100} \mathrm{Os} /{ }^{188} \mathrm{Os}(\lambda)=$ const. for wavelength tuning and mass fractionation correction, the ${ }^{192} \mathrm{Os} /{ }^{188} \mathrm{Os}$ ratio was measured in several different experiments with a reproducibility and accuracy exceeding $0.3 \%{ }^{18}$ In Table 1 we show the mass fractionation per mass unit, $\alpha$, obtained from experiments on different Os resonance transitions. The value of $\alpha$ was calculated assuming a power law: $f(\alpha)=(1+\alpha)^{\mathrm{n}}$. Each value represents an average of several experiments. For zero detuning the mass fractionation factors in each sequence agree to better than $0.4 \%$ and cannot be distinguished within the accuracy of the experiment, demonstrating that a simple mass fractionation law is in effect. For a laser detuning of $0.16 \mathrm{~cm}^{-1}$ differences in the $\alpha$-values are observed which are larger than the experimental error. The detuned $\alpha$-values do not agree for the two transitions because of the large difference in the oscillator strengths of the two transitions, i.e., 0.003 for $J=5$ as compared to 0.3 for $\mathrm{J}=4$. As the mass fractionation also depends on the exact position of the laser beam in the ion source, some variation in $\alpha$ can be expected between different experiments unless the laser beam alignment is very reproducible. 
Table 1. Mass fractionation $\alpha(\% / a m u)$ for even Os isotope ratios

\begin{tabular}{lccc}
$\begin{array}{c}\text { Detuning } \\
\left(\mathrm{cm}^{-1)}\right.\end{array}$ & ${ }^{190} \mathrm{Os} /{ }^{188} \mathrm{Os}$ & ${ }^{192} \mathrm{Os} /{ }^{188} \mathrm{Os}$ & ${ }^{192} \mathrm{Os} /{ }^{190} \mathrm{Os}$ \\
\hline 0 & 6.0 & 7.0 & 8.0 \\
0.16 & 7.7 & 6.3 & 8.2 \\
\hline 0 & 4.0 & 7.0 & 6.0 \\
0.16 & 2.0 & 2.8 & 3.7 \\
\hline
\end{tabular}

${ }^{190} \mathrm{Os} /{ }^{188} \mathrm{Os}$ used for laser wavelength tuning; standard Os isotope ratios from ref. 18. Center of interval with ${ }^{190} \mathrm{Os} /{ }^{188} \mathrm{Os}(\lambda)=$ const. was identified with zero detuning. First row: $\Delta \mathrm{J}=+1$ transition, 39405.9 $\mathrm{cm}^{-1}$. Second row: $\Delta \mathrm{J}=0$ transition, $40361.9 \mathrm{~cm}^{-1}$.

Optical isotope shifts in Ti are much larger than those observed in Os and have a different sign ${ }^{15}$ due to the approach to a closed neutron shell $(\mathrm{n}=28)$ in ${ }^{50} \mathrm{Ti}$. In Fig. 2 the ${ }^{50} \mathrm{Ti} /{ }^{46} \mathrm{Ti}$ ratio as a function of laser detuning is shown together with the ion signal at the individual masses. In this case $\Delta \omega_{L} / \Delta T=3$ and it is apparent that the correct ${ }^{50} \mathrm{Ti} /{ }^{46} \mathrm{Ti}$ ratio cannot be measured at a single wavelength. The ratio of the ion signals measured at the maxima of the individual isotopes should be free of wavelength effects and represent the purely mass fractionated value of that isotope ratio. For other resonances with smaller $\Delta \mathrm{T}$ we obtained ${ }^{50} \mathrm{Ti} /{ }^{46} \mathrm{Ti}(\lambda)=$ const. Very good agreement between isotope ratios measured by tuning the laser to achieve ${ }^{50} \mathrm{Ti} /{ }^{46} \mathrm{Ti}(\lambda)=$ const. and those obtained from the ratio of the maximum signals at masses 50 and 46 justifying our identification of $R_{M}^{i j}(\lambda)=$ const. as the value of the purely mass fractionated isotope ratio. The average values of ${ }^{50} \mathrm{Ti} /{ }^{46} \mathrm{Ti}$ and ${ }^{48} \mathrm{Ti} /{ }^{46} \mathrm{Ti}$ obtained from a series of experiments, including different resonance transitions, are shown in Table 2. The data show that $\alpha$ is constant, independent of mass, and that the corrected ${ }^{48} \mathrm{Ti} /{ }^{46} \mathrm{Ti}$ ratio is indistinguishable from the values of Niederer et al. ${ }^{20}$

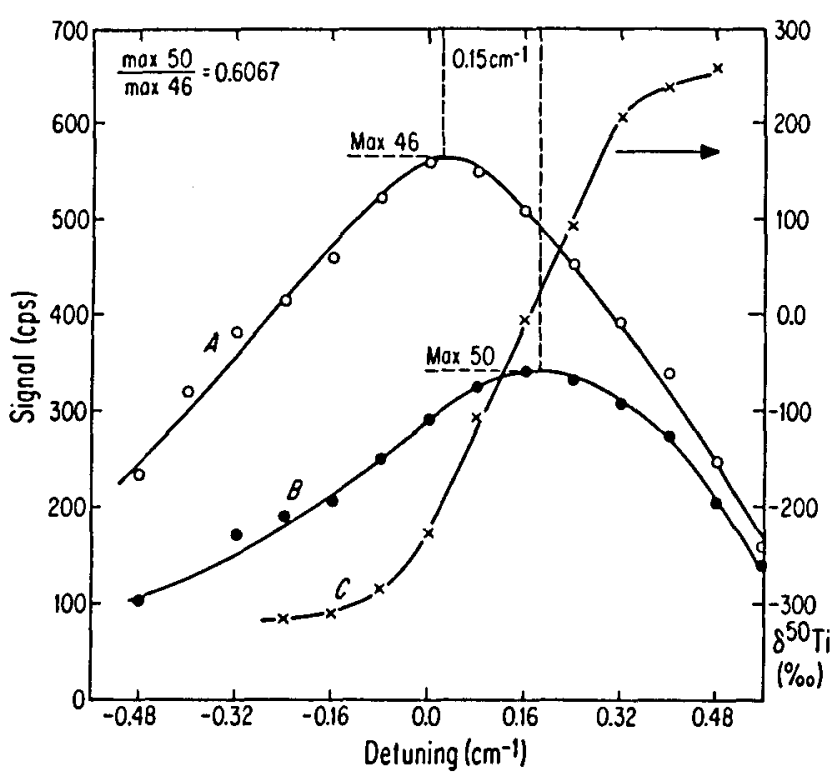

Figure 2. Wavelength dependence of the $\mathrm{Ti}$ ion signal at masses 46 and 50 , curves A and B respectively, shown on the left hand ordinate, and of $\delta^{50} \mathrm{Ti}$ curve $\mathrm{C}$ right hand ordinate; $1 \%<1 \sigma<$ $1.5 \% .39715 .5 \mathrm{~cm}^{-1}, \mathrm{~J}=3$ resonance state. Reference isotopic ratios from Niederer et al. (20). 
Table 2. Ti isotope ratios measured in the region ${ }^{50} \mathrm{Ti} /{ }^{46} \mathrm{Ti}(\lambda)=$ constant

\begin{tabular}{lccc}
\hline & $\begin{array}{c}\text { Measured } \\
\text { Ratio }^{1}\end{array}$ & $\begin{array}{c}\text { Standard } \\
\text { Ratio }\end{array}$ & $\alpha(\%$ omu $)$ \\
\hline${ }^{50} \mathrm{Ti} /{ }^{46} \mathrm{Ti}$ & $0.605(0.007)$ & 0.668 & 25 \\
${ }^{48} \mathrm{Ti} /{ }^{46} \mathrm{Ti}$ & $8.785(0.10)$ & 9.215 & 24 \\
\hline
\end{tabular}

${ }^{1}$ Numbers in parenteses are $\pm 1 \sigma$ errors.

\section{DYNAMIC EFFECTS}

Resonance ionization is a dynamic process which is described by the detailed time evolution of the atomic system interacting with the laser field. ${ }^{3}$ The observation of quantum beats in photoionization ${ }^{21}$ or in the angular distribution of photoelectrons ${ }^{22}$ resulting from a coherent superposition of HFS states gave early evidence of the importance of HFS structure in the resonant excitation-ionization process. ${ }^{23}$ The importance of these effects for isotope ratio measurements with laser ion sources has been realized only recently $y^{11,22,13}$ following the observation of anomalous odd-even isotope ratios measured on a $\Delta \mathrm{J}=+1$ transition. ${ }^{8}$ Selection rule arguments provided no evidence that odd isotope enhancement should be observed for that transition. Compared to the ionization of single fine structure level, the presence of HFS changes the dynamics of the excitation-ionization process in two ways which may be described as the coherent and incoherent cases. Both limiting situations may result in equal ionization probabilities for odd and even isotopes. The connection between the two cases was recently discussed by Payne et al. ${ }^{13}$ We assume here that the laser bandwidth, $\Delta \omega_{L}$, is larger then the hyperfine splitting, $\Delta \omega_{\text {HFS }}$.

If the laser pulse duration $t_{p}$ is much smaller then the hyperfine coupling time $\Delta \omega_{\text {HFS }}^{-1}$, a coherent superposition of HFS levels is generated from which ionization occurs. In this case ionization proceeds as if HFS structure were absent ${ }^{11,13,24}$ with half the atomic population in the ground state and the other half in the excited state. For times short compared to the HFS coupling period the individual angular momenta of the nucleus and the electrons are still good quantum numbers and no odd-even selectivity is expected. In addition there is a second order interference term ${ }^{22,25}$ arising from the coupling of two different HFS levels to the same continuum state which can cause quantum beats in the total photoionization. The importance of this term has been analyzed by Lampropoulos et al. ${ }^{22}$ and Payne et al. ${ }^{13}$

If $t_{p}>\omega_{H F S}^{-1}$ the coherence between the HFS levels is destroyed and ionization proceeds via independent HFS states. This situation is depicted in Fig. 3 which shows the linkage diagram for a transition from a $\mathrm{J}=0$ state to $\mathrm{J}=1$ state for isotopes with nuclear spin $\mathrm{I}=1 / 2$ and with $\mathrm{I}=0$ (even isotope). Due to the larger excited state degeneracy, two thirds of the atomic population is in the excited state for $I=$ $1 / 2$ compared to one half for the isotope with $I=0$. If the ionization is not saturated, i.e., $R_{I} \times t_{p}<1$, large differences in the ion yield for odd and even isotopes with an enhancement of the odd isotope will result. In addition differences in the photoionization cross sections of the odd and even isotopes ${ }^{11}$ arise from angular momentum factors. The odd isotope enhancement will diminish as saturation of the ionization is approached. If $\mathrm{R}_{\mathrm{I}}>\omega_{H F S}^{-1}$, ionization proceeds again from a coherent superposition with equal ionization probabilities for odd and even isotopes. For a laser pulse duration of $10 \mathrm{~ns}$ both situations will be encountered in practical RIMS measurements, i.e., the coherent and incoherent cases. The use of lasers with $t_{p}<0.1 \mathrm{~ns}$ has been suggested as one approach to avoid isotopic selectivity. ${ }^{11}$ For most systems, 
(a)

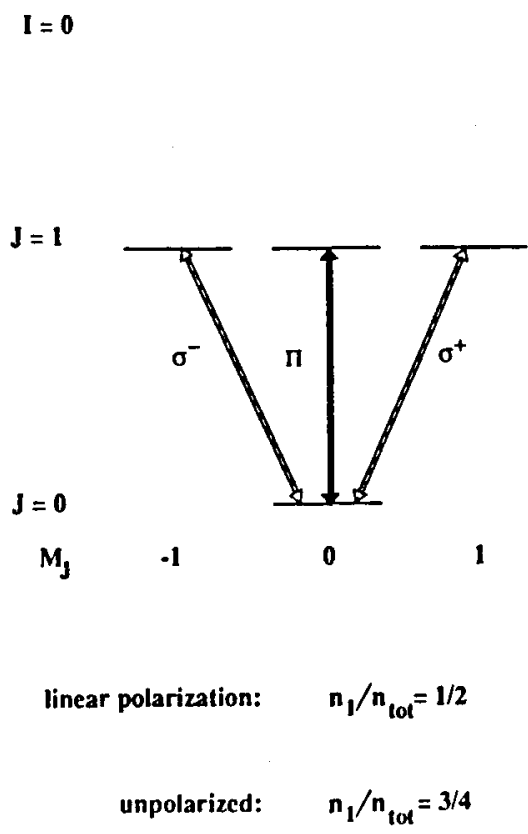

(b)

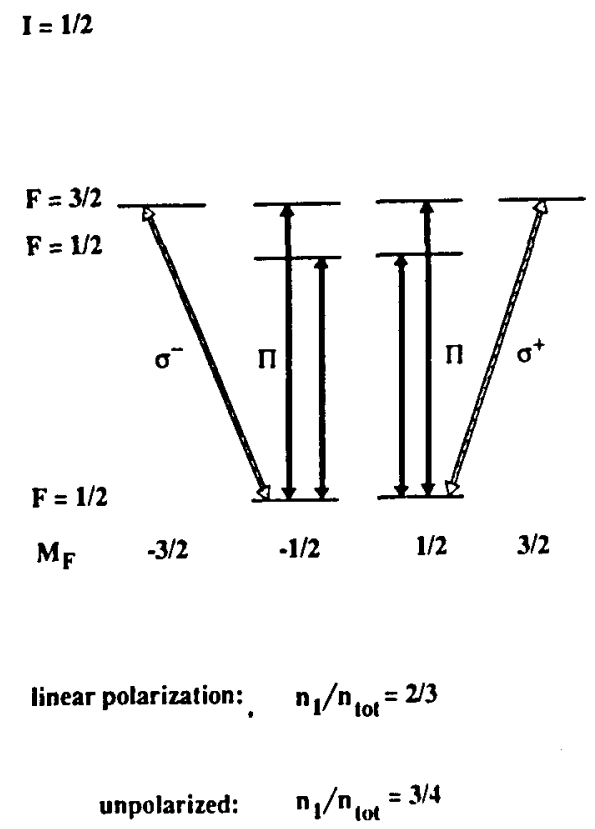

Figure 3. Linkage diagram for a $\Delta J=+1$ transition. (a) nuclear spin $I=0$, (b) $I=1 / 2$

however, saturation of the ionization in such a short time interval requires large laser intensities with a concommitant increase in the off-resonant ionization background, particularly for sputter atom sources. Detuning of the excitation laser by an amount larger than $\Delta \omega_{\mathrm{HFS}}$ from the intermediate resonance has been suggested by Payne $e t$ al. ${ }^{13}$ to avoid isotopic selectivity. In the case $\mathrm{R}_{\mathrm{I}} \times \mathrm{t}_{\mathrm{P}}>1$ and assuming $\mathrm{t}_{\mathrm{P}}>\omega_{\text {HFS }}^{-1}$, the ionization yield will be the same for odd and even isotopes in the high intensity region of the laser beam, while low intensity regions with $R_{1} \times t_{P}<1$ contribute different odd and even ionization yields to the total ion signal. ${ }^{13}$ The size of the effect depends on the focusing conditions and the details of the ion extraction. To minimize this effect the confocal parameter of the focussed laser beam should be larger than the spatial extent of the ionization volume. Using an ionization laser with a larger beam waist than the excitation laser will also reduce the influence of edge effects on odd-even isotopic ratios.

Our experiments on $\Delta \mathrm{J}=+1$ transitions in Os and Ti were carried out in the incoherent regime with $\Omega_{R}>R_{I}$, so that the pulse duration determines the time scale of the ionization process. In Table 3 we show odd- even isotope ratios for Os and $\mathrm{Ti}$. The ratios have been corrected for mass fractionation as described above. The individual ratios represent averages of several experiments, including realignments of the optical set up and repeated wavelength tuning. It is seen that under saturating conditions the odd-even isotope ratios for both $\mathrm{Os}$ and $\mathrm{Ti}$ are indistinguishable from the true abundance ratio in the sample. 
Table 3. Even-odd effects in Os and $\mathrm{Ti}$ isotope ratios for a $\Delta \mathrm{J}=+1$ transistion.

\begin{tabular}{lcccc}
\hline $\begin{array}{l}\text { Resonance } \\
\text { State }\left(\mathrm{cm}^{-1)}\right.\end{array}$ & $\begin{array}{c}\delta^{47} \mathrm{Ti}^{1} \\
(\% 0)\end{array}$ & $\begin{array}{c}\delta^{49} \mathrm{Ti}^{1} \\
(\% 0)\end{array}$ & $\begin{array}{c}\delta^{189} \mathrm{Os}^{2} \\
(\% 0)\end{array}$ & sat \\
39405.9 & & $-3.4(5)$ & sade \\
38544.4 & $-14(12)$ & $+2.8(11)$ & sat \\
37851.5 & $+35(11)$ & $+4.6(11)$ & nsat \\
38159.5 & $+60(15)$ & $+144(23)$ & & sat \\
\hline
\end{tabular}

${ }^{1}$ Deviation in permil of measured ratio from standard value in ref. 20 . Reference isotope ${ }^{46} \mathrm{Ti}$.

Numbers in parentheses are $\pm 1 \sigma$ error.

${ }^{2}$ Deviations in permil of measured ratio from standard values in ref. 18. Reference isotope ${ }^{190}$ Os.

${ }^{3}$ Sat indicates saturation of ionization; nsat indicates that the ionization was not saturated.

These results differ from those obtained by Fassett and Walker for $O s^{8}$ and Spiegel et al. for $\mathrm{Ti}^{10}$ where enrichments of $3 \%$ for ${ }^{189} \mathrm{Os}$ and $40 \%$ for ${ }^{49} \mathrm{Ti}$ have been observed. Under nonsaturating conditions a significant increase in the Ti odd isotope abundances is observed. The higher value of $\delta^{49} \mathrm{Ti}$ compared to that for $\delta^{47} \mathrm{Ti}$ may be due to the larger number of excited state components of ${ }^{49} \mathrm{Ti}(\mathrm{I}=7 / 2)$ compared to ${ }^{47} \mathrm{Ti}(\mathrm{I}=5 / 2)$. In Fig. 4 the ${ }^{189} \mathrm{Os} /{ }^{188} \mathrm{Os}$ and ${ }^{189} \mathrm{Os} /{ }^{190} \mathrm{Os}$ isotope ratios as a function of laser pulse energy are shown for the $39406 \mathrm{~cm}^{-1} \Delta \mathrm{J}=+1$ transition. The maximum power density was $5 \times 10^{7} \mathrm{Watt} / \mathrm{cm}^{2}$; the arrow indicates the onset of ionization saturation. In this experiment the odd-even isotope ratios are constant until the laser pulse energy is reduced to less than half the saturation value. Following the arguments outlined above, an enrichment of the odd isotope is expected with decreasing degree of ionization saturation. An upper limit for $\Delta \omega_{\mathrm{HFs}}$ of $0.34 \mathrm{~cm}^{-1}$ for this transition was obtained compared to a laser bandwidth of $0.4 \mathrm{~cm}^{-1}$. We calculate that the discrete resonance for an HFS component with an oscillator strength as low as $\mathbf{0 . 0 0 1}$ and which is detuned one laser bandwidth from line center should be saturated at the lowest energies used in this experiment. The oscillator strength of the transition was estimated as 0.003 from the power broadened width of the ionization lineshape. With these values the constancy of the odd-even ratios for laser energies larger than half the saturation energy cannot be explained by laser bandwidth or tuning effects. This is also supported by the symmetry in the ${ }^{189} \mathrm{Os} /{ }^{188} \mathrm{Os}$ and ${ }^{189} \mathrm{Os} /{ }^{190} \mathrm{Os}$ isotope ratios.

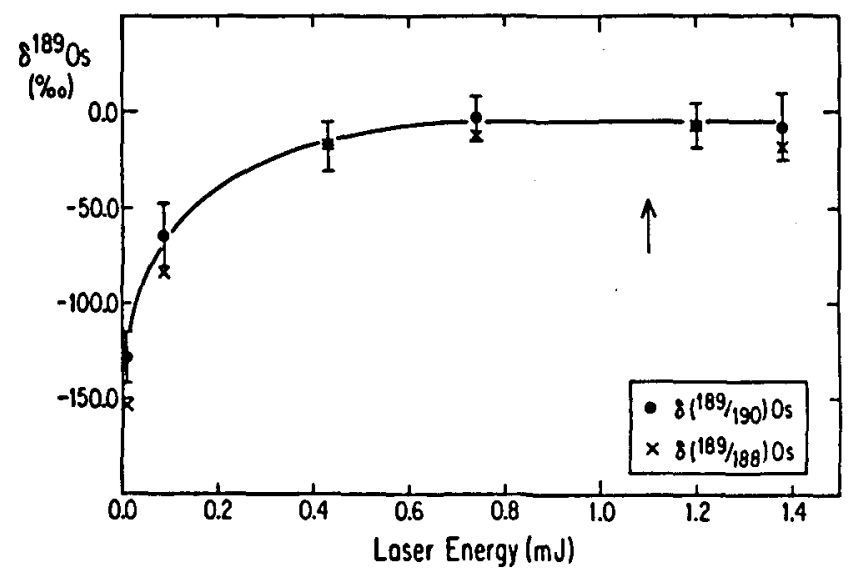

Figure 4. Variation of the ${ }^{189} \mathrm{Os} /{ }^{190} \mathrm{Os}$ (filled circles) and ${ }^{189} \mathrm{Os} /{ }^{188} \mathrm{Os}$ (open circles) as a function of laser energy per pulse for the 39047. $\mathrm{cm}^{-1} \Delta \mathrm{J}=+1$ transition. Isotope ratios are expressed in $\delta$-values with regard to standard values from Creaser et al. ${ }^{18}$ 


\section{SELECTION RULE AND POLARIZATION EFFECTS}

Selection rule effects are best visualized with the linkage diagram shown in Fig. 4 read from the top down. In this case it represents a transition from a $\mathrm{J}=1$ to a $\mathrm{J}=0$ state. From the $\Delta \mathrm{M}_{\mathrm{J}, \mathrm{F}}=0$ selection rule for linearly polarized light two thirds of the even isotope $(I=0)$ ground state population does not participate in the transition, while for the odd isotopes $(I=1 / 2)$ this number is reduced to one third, producing an increase of the odd isotope ion signal. Selection rules are usually considered as static, so that the odd isotope enrichment could be precisely calculated by counting levels as described above. ${ }^{26}$ However, selection rule effects can acquire a dynamic character for two different reasons. As described above for $t_{p}>\omega_{H F S}^{-1}$, the excitation proceeds as if HFS structure were absent. In this case no odd-even effect should be present on $\Delta \mathrm{J}=0$ or $\Delta \mathrm{J}=-1$ transitions. Thus the deviation of odd-even isotope ratios from their standard values for these transitions can be very different depending on the $t_{p}>\omega_{H F S}^{-1}$ ratio when measured with linearly polarized light. In the second case and considering $t_{p}>\omega_{H F S}^{-1}$, a dynamic aspect is introduced by the fact that in many cases the laser radiation is not perfectly polarized. Isotope ratios will then depend on the detailed excitation and ionization characteristics of ground state $\mathrm{M}_{\mathrm{J}}$ or $\mathrm{M}_{\mathrm{F}}$ components which interact with the major and minor polarization components. ${ }^{14}$ The odd-even isotope ratios will then depend on the polarization characteristics, intensity and beam parameters of the laser and also on the ion optical extraction conditions.

We performed a series of odd-even ratio measurements for $\Delta \mathrm{J}=0$ and $\Delta \mathrm{J}=-1$ transitions in Os and Ti in the regime $t_{p}>\omega_{H F S}^{-1}$ to study the influence of depolarization and focussing on odd-even isotopic ratios. The experiments were performed under saturating conditions (ionization) with $R_{\mathrm{I}}<\Delta \omega_{\text {HFs. }}$. Some degree of depolarization was generated by passing the laser beam through a quartz window exposed to localized stress, which created a random amount of birefringence. From the results shown in Table $4 \mathrm{a}$ it is evident that the odd-even effect is greatly reduced with increasing depolarization of the laser beam. For the $\Delta J=$ 0 and $\Delta \mathrm{J}=-1$ transitions in Ti and Os, respectively, no odd isotope enhancement is observed. From selection rule arguments and with linearly polarized light we calculate odd isotope enrichments of $21.5 \%$ and $12.5 \%$ for ${ }^{189} \mathrm{Os}$ on the $\Delta \mathrm{J}=-1$ and $\Delta \mathrm{J}=0$ transitions, respectively, and a $22.5 \%$ enrichment of ${ }^{47} \mathrm{Ti}$ for the $\Delta \mathrm{J}=0$ transition. The residual $3.6 \%$ odd isotope enhancement on the $\Delta \mathrm{J}=0$ transition in Os appears unusual considering that the latter resonance has an oscillator strength of 0.3 compared to $\sim 0.003$ for the $\Delta \mathrm{J}=-1$ transition. A rather small admixture of depolarization should saturate the otherwise forbidden $\Delta \mathrm{M}_{\mathrm{J}, \mathrm{F}}=+1,-1$ transitions. This resonance was initially chosen because odd-even effects were expected to be completely absent for a small degree of depolarization. The effect of focussing on odd-even ratios for the same degree of depolarization is shown in Table $4 \mathrm{~b}$. It is interesting to note that at least for $\Delta \mathrm{J}=0$ transition in Os, the Rabi frequency is much larger than the hyperfine coupling period. However, as seen from the isotope ratios, the ionization does not proceed as if HFS structure were absent. The atom frequently returns to the exicted state before it is ionized so that HFS coupling can act. This aspect was mentioned by Shore and Johnson ${ }^{25}$ and later by Lambropoulos and Lyras. ${ }^{11}$ In order to demonstrate the effect of a small admixture of depolarized light, which can drive $\Delta \mathrm{M}_{\mathrm{J}, \mathrm{F}}=+1$ and -1 transitions, as seen in the absence of odd-even effects for the $\Delta \mathrm{J}=-1$ transition for $P_{\mathrm{ell}}=27$, we performed a rate equation calculation of the spatial distribution of the steady state saturation behavior of the discrete resonance for the different $\Delta \mathrm{M}_{\mathrm{J}, \mathrm{F}}=0,+1$ and -1 transitions. It was assumed that the linearly polarized component is the major polarization component with a small admixture of depolarization expressed by $\mathrm{P}_{\mathrm{ell}}$. In addition, the ionization probability of the different ground state angular momentum states was calculated from which the odd isotope enrichment can be inferred. In Fig. 5, results are shown for two different focussing conditions calculated with the atomic parameters of the Os $\Delta J=-1$ transition. The agreement between calculated and measured isotope ratios is quite good. The model calculations also demonstrate that isotope 

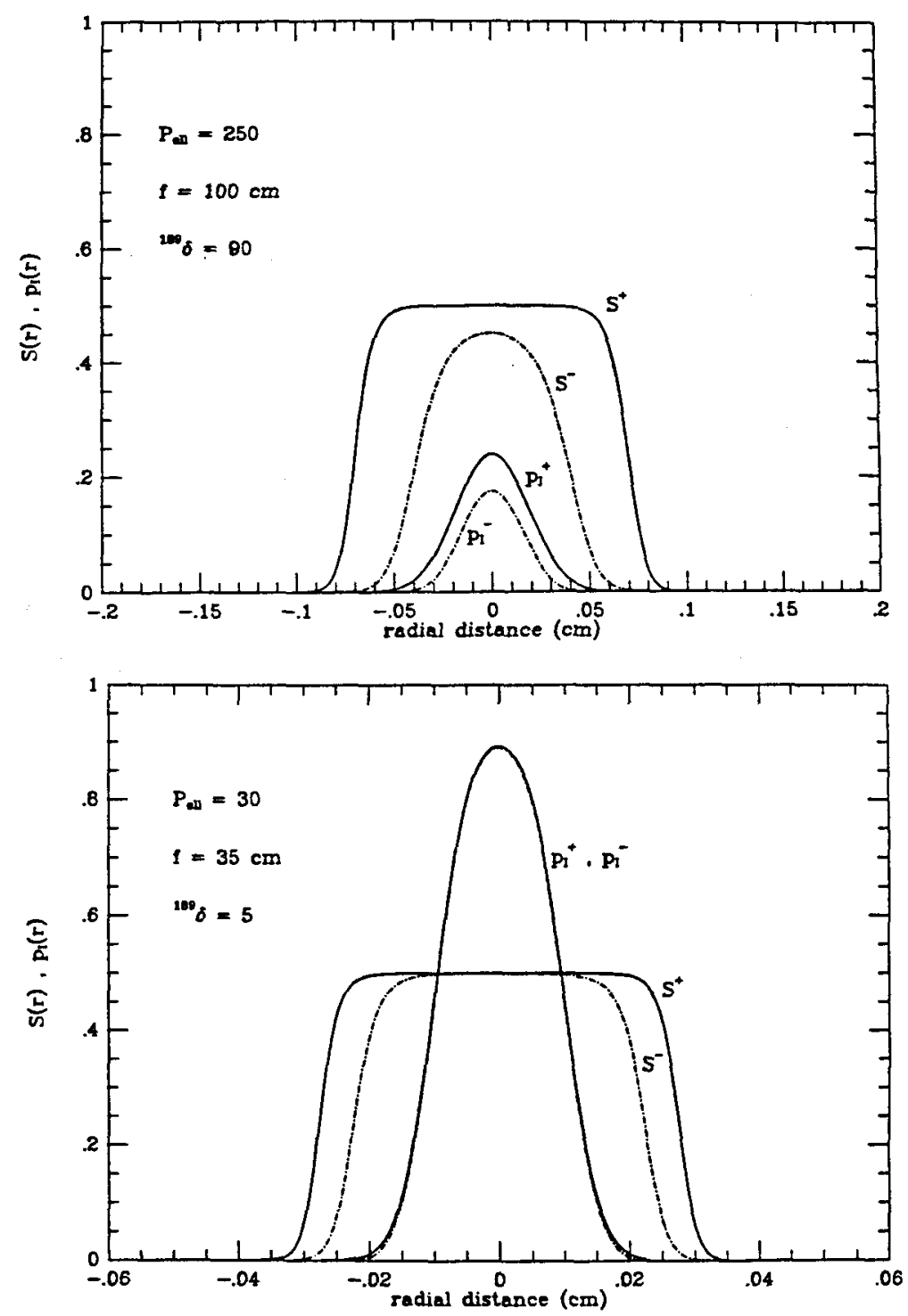

Figure 5. Radial dependence of the steady state saturation $S(r)$ and ionization probability $P_{1}(r)$ for two $M_{3}$ components of the $\Delta \mathrm{J}=-1$ transition in Os $\left(39383 \mathrm{~cm}^{-1}\right)$ which interact with the major " + " and minor "-" polarization component.

ratios measured with multistep ionization schemes on $\Delta \mathrm{J}=0$ or $\Delta \mathrm{J}=-1$ transitions can depend critically on laser beam overlap, in particular with non-colinear beam propagation. This can have significant effects on the reproducibility of measured isotope ratios. The polarization scrambling experiments have shown that the odd isotope enrichment can be reduced to the $3 \%$ level or lower as a function of depolarization of the laser beam and the focussing conditions. This can considerably enhance the versatility of available RIMS schemes. It also appears from the linkage diagram in Fig. 2 that polarization scrambling can be used to reduce the dynamic odd-even effects on $\Delta \mathrm{J}=+1$ transitions. 
Table 4a. Odd-even isotope ratios for Os and $\mathrm{Ti}$ for $\Delta \mathrm{J}=0$ and -1 transitions for two different degrees of depolarization of the laser radiation.

$$
\mathrm{P}_{\mathrm{ell}}=220^{1} \quad \mathrm{P}_{\mathrm{ell}}=27^{1}
$$

\begin{tabular}{lcc}
$\delta^{47} \mathrm{Ti}(\% 0)^{2}$ & $+137(10)$ & $+2.7(10),+16(15)$ \\
$\delta^{189} \mathrm{Os}(\%)^{3}$ & $+57(8)$ & $-1.3(13)$ \\
$\delta^{189} \mathrm{Os}(\% 0)^{3}$ & $+89(10)$ & $+36(8)$ \\
\hline
\end{tabular}

${ }^{1} \mathbf{P}_{\text {ell }}$ is the maximum intensity ratio for two orthogonal positions of a linear polarizer measured after transmission of the mass spectrometer. Strong focussing conditions $(\mathrm{f}=35 \mathrm{~cm}$ ).

${ }^{2}$ Deviation in permil from ${ }^{42 \wedge} \mathrm{Ti} /{ }^{46} \mathrm{Ti}$ ratio in ref. 20.

${ }^{3}$ Deviation in permil from ${ }^{189} \mathrm{Os} /{ }^{190} \mathrm{Os}$ ratio in ref. 18 . Numbers in parentheses are $\pm 1 \sigma$ error.

Table $4 \mathrm{~b}$. Dependence of $\delta^{189} \mathrm{Os}$ on the focussing conditions for $\Delta \mathrm{J}=-1$ and 0 transitions and $P_{\text {ell }}=27$.

\begin{tabular}{lccc}
\hline & $\Delta \mathrm{J}^{1}$ & $\mathrm{f}=100 \mathrm{~cm}$ & $\mathrm{f}=35 \mathrm{~cm}$ \\
\hline$\delta^{189} \mathrm{Os}(\% 0)^{2}$ & -1 & $74(11)$ & $-1.3(13)$ \\
& 0 & $57(16)$ & $+36(8)$ \\
\hline
\end{tabular}

${ }^{1} \Delta \mathrm{J}=-1$ corresponds to the $39382.9 \mathrm{~cm}^{-1}$ resonance state, $\Delta \mathrm{J}=\mathrm{O}$ corresponds to the $403961.9 \mathrm{~cm}^{-1}$ resonance state. Numbers in parentheses are $\pm 1 \sigma$ error.

${ }^{2}$ Deviation in permil from ${ }^{189} \mathrm{Os} /{ }^{190}$ Os ratio in ref. 18 .

\section{SUMMARY}

Although rather straightforward from an experimental point of view, the application of RIMS to the precise measurement of isotopic abundances is quite complex because of the many physical processes that may affect measured isotope ratios. We have shown that under certain circumstances, such as a transition with $\Delta \mathrm{J}=+\mathrm{I}$, strong saturation of the resonance ionization process, confocal parameter of the laser beam larger then the ion extraction region or for depolarization of the laser beam, isotope abundances including oddeven ratios, can be measured, which differ from the true isotopic abundances only by pure mass fractionation. A technique employing the wavelength dependence of even isotope ratios as an internal wavelength standard was developed to permit accurate and reproducible wavelength adjustment of the laser radiation. Using this technique with laser parameters satisfying the principles outlined above, we obtained reproducible measurements of Os and $\mathrm{Ti}$ isotopic abundances. Even-even isotope ratios exhibit only mass fractionation, i.e., no wavelength dependence, and even-odd isotope shifts are smaller than $2 \%$. These results demonstrate for the first time that under selected conditions RIMS measurements can provide isotopic ratios unaffected by the photoionization process itself.

The complexity of the ionization process, including hyperfine structure, necessitates studies of the effect of laser parameters and ion optical extraction conditions on odd-even isotope ratios for each ionization scheme. It appears important to investigate further the level of accuracy that can be obtained in the measurement of odd-even isotope ratios. 


\section{ACKNOWLEDGMENTS}

This work was supported by DOE grant DE FG03-88ER-13851 and NSF grant EAR-8816936 to G. J. Wasserburg. The laser system was obtained through support from the Packard and Sloan Foundations and NASA grant NAGW-1955 to G. A. Blake. Division Contribution 5113(767).

\section{REFERENCES}

(1) J.E. Parks, N.T. Spear, D.W. Beekman and L.J. Moore, Resonance lonization Spectroscopy 1988, T.B. Lucatorto and J.E. Parks (Eds.), Institut of Physics Conf. Series 94 p. 197, Bristol, UK, 1988

(2) M.J. Pellin, C.E. Young, W.F. Caraway, J.W. Burnett, B. Jorgensen, E.L. Schweitzer and D.M. Gruen, Nucl. Instrum. Method. B 18 (1987) 446

(3) G.S. Hurst, M.G. Payne S.D. Kramer and J.P. Young, Rev. of Mod. Phys. 51 (1979) 767

(4) R.S. Lewis, S. Amari and E. Anders, Nature 348 (1990) 293

(5) J. Stone, I.D. Hutcheon, S.E. Epstein and G.J. Wasserburg, Earth and Planetary Science Lett. 107, 570-581, (1991).

(6) R.J. Walker and J.D. Fassett, Anal. Chem., 58 (1986) 2393

(7) Y.P. Young, R.W. Shaw, D.E. Goeringer and D.H. Smith, Resonance lonization Spectroscopy 1988, T.B. Lucatorto and J.E. Parks (Eds.), Institute of Physics Conf. Series 94, p.367, Bristol UK, 1988

(8) W.M. Fairbank Jr., M.T. Spear, J.E. Parks and J.M.R. Hutchinson, Phys.Rev. A 40(1989)2195

(9) C Hayman, B. Comaskey, M. Johnson and W. Worden, SPIE Proceedings, 912 (1988) 200

(10) D.R. Spiegel, M. J. Pellin, W.F. Callaway, J.W. Bumett, S.R. Coon, C.E. Young, D.M. Gruen and R.N. Clayton, Proceedings Lunar and Planetary Science Conf 22 (1991)p.1303

(11) P. Lambropoulos and A. Lyras, Phys. Rev. A 40 (1989) 2199

(12) A. Lyras, B. Zorinan and P. Lambropoulos, Phys.Rev. A 42 (1990) 543

(13) M.G Payne, S.L Allman and J.E. Parks Spectrochemica Acta 46B (1991) 1439

(14) R.K. Wunderlich, I.D. Hutcheon, G.J. Wasserburg and G. A. Blake, submitted Int. J. of Mass Spectrom. and Ion Proc.

(15) R.K. Wunderlich, G.J. Wasserburg, I.D. Hutcheon, and G. A. Blake, submitted Spectrochemica Acta

(16) G.J. Wasserburg, J.B. Jacobson, D.J. DePaolo, M.T. Colloch and T. Wen, Geochim. Cosmochim. Acta 45 (1971) 2311 
(17) S.A. Hart and A. Zindler, Int.J. Mass Spectrom. and Ion. Proc. 89 (1989) 282

(18) R.A. Creaser, D.A. Papanastassiou and G.J. Wasserburg, Geochim. Cosmochim. Acta 55 (1991) 597

(19) C.M. Miller, B.L. Fearey, B.A. Palmer and N.S. Nogar, Resonance lonization Spectroscopy, 1988, T.B. Lucatorto and J.E. Parks (Eds.), Institute of Physics Conf. Series 94, p.297, Bristol UK, 1988

(20) F.R. Neederer, D.A. Papanastassiou and G.J. Waserburg Geochim. Cosmochim. Acta 45 (1981) 1017

(21) T.W. Ducas, M.G. Littman and M.L. Zimmerman, Phys. Rev. Lett. 35 (1975) 1752

(22) G. Leuchs, S.J. Smith, E. Khawaja and H. Walther, Opt. Commun. 31 (1979) 313

(23) A.T. Georges and P. Lambropoulos, Phys. Rev. A 18 (1978) 1072

(24) B.W. Shore and M.A. Johnson Phys. Rev. A23 (1981) 1608

(25) P.L. Knight, Opt. Commun. 31 (1978) 148

(26) L.C. Balling and J.J. Wright, Appl. Phys. Lett.29 (1976) 411 